\title{
Parasitismo por Calodium hepaticum em dois cães
}

\author{
Calodium hepaticum parasitism in two dogs \\ Heloísa Einloft PalmaI Paula Cristina Basso ${ }^{\mathrm{II}}$ Anne Santos do Amaral ${ }^{\mathrm{II}}$ \\ Ana Paula Silva ${ }^{\text {IV }}$ Cândido Fontoura Silva ${ }^{\text {III }}$
}

\section{- NOTA -}

\section{RESUMO}

Calodium hepaticum já foi relatado parasitando o parênquima hepático de diversas espécies de mamíferos, porém é infrequente em cães. O presente artigo tem o objetivo de descrever dois casos de capilariose hepática em cães, diagnosticados em um período de um mês na região de Santa Maria, Rio Grande do Sul, Brasil. O primeiro cão apresentava sintomatologia clínica de insuficiência cardíaca, e ovos de Calodium hepaticum foram encontrados ocasionalmente no exame histopatológico do fígado. O segundo animal apresentava mucosas ictéricas e ascite. No hemograma, ficou evidente a presença de anemia arregenerativa e, na avaliação de bioquímica sérica, percebeu-se aumento de fosfatase alcalina, alanina aminotransferase e hipoalbuminemia. A análise do liquido cavitário foi compatível com transudato modificado. No exame histopatológico, foram observados numerosos ovos bioperculados de Calodium hepaticum, dispostos aleatoriamente pelo parênquima hepático, com reação granulomatosa e fibrosa adjacente aos ovos. A existência de maior número de cães infectados demonstra a necessidade de controle da população de roedores.

Palavras-chave: capilariose, nematóide, insuficiência hepática, cão.

\section{ABSTRACT}

Calodium hepaticum has been reported as a parasite of the hepatic parenchyma of many mammal species, but it is not frequent in dogs. The present article aimed to describe two cases of hepatic capillariasis in dogs, diagnosed in a period of one month, in e Santa Maria city, Rio Grande do Sul state, Brazil . The first dog presented clinical sintomathology of cardiac insufficiency and Calodium hepaticum eggs were occasionally found by histopathological examination of the liver. The second animal presented icteric mucous membranes and ascites. The hemogram revealed non-regenerative anaemia and in the biochemistry serum were observed elevation of alkaline phosphatase, alanina aminotransferase and hypoalbuminemia. The cavitary liquid analysis was compatible with modified transudate. In the histopathological examination, numerous bioperculated eggs of Calodium hepaticum were observed aleatorily distributed in the hepatic parenchyma, with granulomatous and fibrous reaction adjacent to the eggs. The occurrence of two cases in a short period of time suggests that other dogs may be infected and requires a rodent population control.

Key words: capillariasis, parasites, hepatic insufficiency, dog.

Calodium hepaticum, outrora denominado Capillaria hepatica, é um nematódeo pertencente à família Trichuroidae, sendo relatado parasitando o parênquima hepático de mais de 40 espécies de mamíferos, tais como cães, gatos, suínos, macacos e coelhos, sendo mais comumente encontrado em roedores, especialmente nos ratos (SAWAMURA et al., 1999; SANTOS et al., 2001; RUAS et al., 2003). A alta prevalência e baixa intensidade de infecção em Rattus norvegicus sugerem que essa espécie seja o hospedeiro primário de C. hepaticum (ILHA \& BARROS, 2000).

${ }^{\mathrm{I} C u r s o}$ de Medicina Veterinária, Universidade Federal de Santa Maria (UFSM), RS, Brasil.

IIPrograma de Pós-graduação em Medicina Veterinária, UFSM, 97105-900, Santa Maria, RS, Brasil. E-mail: paula.basso@bol.com.br. Autor para correspondência.

IIIDepartamento de Clínica de Pequenos Animais (DCPA), UFSM, Santa Maria, RS, Brasil.

${ }^{\text {IV}}$ Programa de Residência em Medicina Veterinária, UFSM, Santa Maria, RS, Brasil. 
A infecção por $\boldsymbol{C}$. hepaticum ocorre por ingestão dos ovos infectantes liberados do fígado, após morte e decomposição do hospedeiro, por canibalismo ou por predação (FARHANG-AZAD \& SCHLITTER, 1978; REDROBE \& PATTERSON-KANE, 2005). O hábito de predação em animais domésticos de rua contribui para a disseminação de ovos, constituindo fonte de infecção para eles, para outros animais e para o homem (ILHA \& BARROS, 2000).

A capilariose hepática é rara em caninos e geralmente é um achado incidental de necropsia. No entanto, ocasionalmente, esses pacientes podem exibir sinais clínicos de insuficiência hepática, como anorexia, vômito, diarréia, icterícia, ascite e distúrbios neurológicos (ILHA \& BARROS, 2000), atribuídos à encefalopatia hepática (TABOADA \& DIMSKI, 1995). O diagnóstico é obtido por meio dos típicos achados histopatológicos de ovos bioperculados no fígado (SOLOMON \& HANDLEY, 1971; SAWAMURA et al., 1999). O tratamento na espécie canina ainda é desconhecido; entretanto, em humanos, têm sido utilizadas drogas antinematoides de amplo espectro, como albendazol e tiabendazol (SAWAMURA et al., 1999).

Este trabalho tem o objetivo de relatar a ocorrência, os sinais clínicos e as lesões macro e histológicas associadas ao parasitismo por $\boldsymbol{C}$. hepaticum em dois cães atendidos em abril de 2008, no Hospital Veterinário da Universidade Federal de Santa Maria(UFSM).

O primeiro cão atendido era um macho, de 13 anos de idade e sem raça definida. Conforme relato do proprietário, o paciente era cardiopata e estava sendo tratado com furosemida, maleato de enalapril e digoxina. Havia recebido superdosagem de digitálico, passando a apresentar vômito bilioso abundante. No exame físico, o paciente apresentava mucosas rosadas, temperatura retal de $38,5^{\circ} \mathrm{C}$ e estado nutricional regular. $\mathrm{Na}$ palpação abdominal, percebeu-se presença de líquido abdominal livre e, na auscultação torácica, ficou evidente abafamento dos sons cardiopulmonares. $\mathrm{O}$ exame radiológico revelou aumento na densidade radiográfica da cavidade torácica, obscurecendo a silhueta cardíaca, imagem compatível com efusão pleural. Posteriormente à drenagem torácica, foi realizada nova avaliação radiológica, na qual foi evidenciada cardiomegalia. O líquido peritoneal foi classificado como transudato modificado, confirmando o diagnóstico de insuficiência cardíaca. O paciente foi internado, passando a receber fluidoterapia e metoclopramida. Em razão da piora do quadro geral, o cão foi submetido à eutanásia.
$\mathrm{Na}$ necropsia, foi observada discreta endocardiose valvar mitral. O fígado apresentava superfície capsular com aspecto reticulado (coloração mesclada de amarelo e vermelho), levemente granular e aspecto arenoso ao corte. $\mathrm{Na}$ histologia foram observados numerosos ovos bioperculados de $\boldsymbol{C}$. hepaticum e em associação a esses ovos havia necrose multifocal, mineralização e fibrose.

O segundo animal era um cão macho, de nove anos de idade e sem raça definida. Apresentava histórico de aumento progressivo de volume abdominal e dificuldade para defecar há uma semana. No exame físico, constataram-se mucosas ictéricas e efusão abdominal. Foram realizados exames complementares de bioquímica sérica, hemograma e análise do líquido peritoneal. As alterações encontradas foram: aumento da fosfatase alcalina $\left(279 \mathrm{UI} \mathrm{L}^{-1}\right)$, hipoalbuminemia $(0,85 \mathrm{~g}$ $\left.\mathrm{dL}^{-1}\right)$, leucocitose por neutrofilia $\left(21.500 \mu \mathrm{L}^{-1}\right)$ e anemia normocítica normocrômica (hematócrito: $21 \%$; VCM: 68,6fL; CHCM: 31,0\%). O líquido peritoneal foi classificado como transudato modificado. O cão permaneceu internado por uma semana sem apresentar melhora clínica, mesmo com a instituição de tratamento de suporte com fluidoterapia, antibiótico (Cefalotina, $30 \mathrm{mg} \mathrm{kg}^{-1}$, IV, TID; Metronidazol, 25 mg kg-1 IV, BID) e diurético (Furosemida, $2 \mathrm{mg} \mathrm{kg}^{-1}$, SC, TID). A pedido do proprietário, foi realizada eutanásia.

$\mathrm{Na}$ avaliação macroscópica do fígado do segundo animal, verificou-se coloração esverdeada e aspecto micronodular na superfície natural e de corte. $\mathrm{Na}$ avaliação histológica, ficou evidente presença de fibrose em ponte e múltiplos nódulos de regeneração de hepatócitos, hiperplasia de ductos biliares e fibrose (Cirrose Hepática). Foram observados numerosos ovos bioperculados de C. hepaticum, dispostos aleatoriamente pelo parênquima hepático, com reação granulomatosa e fibrosa adjacente aos ovos. Estabeleceu-se o diagnóstico de cirrose e capilariose hepática.

Conforme o histórico clínico e laudo histopatológico do cão 1 , a causa da morte não foi o parasitismo por $\boldsymbol{C}$. hepaticum, sendo considerado um achado incidental. Com relação ao segundo cão, não foi possível definir se o animal apresentava cirrose e posteriormente foi infectado pelo parasita ou se o intenso parasitismo incitou a ocorrência de cirrose. As alterações histopatológicas encontradas no último cão condizem com as observadas por SAWAMURA et al. (1999), os quais relataram que a desintegração dos parasitas e liberação dos ovos no fígado provocam necrose hepática, reação inflamatória e fibrose septal, frequentemente tendendo à cirrose. 
A infecção por C. hepaticum não é frequente em cães. Conforme relatado por CERUTI et al. (2001), de aproximadamente 500 cães e gatos necropsiados na Faculdade de Medicina Veterinária de Milão, apenas um cão foi positivo. Esses dados assemelham-se aos observados em um trabalho realizado por ILHA \& BARROS (2000), no Laboratório de Patologia Veterinária da Universidade Federal de Santa Maria, em que, de 3.927 cães e 435 gatos necropsiados, nove cães $(0,23 \%)$ e seis gatos $(1,38 \%)$ apresentaram capilariose hepática. Ressalta-se aqui o fato de os dois casos de capilariose hepática terem sido diagnosticados em um curto espaço de tempo (30 dias), o que diferencia dos trabalhos descritos anteriormente e evidencia uma situação de alerta aos veterinários da região para essa doença.

Em caninos os relatos clínicos são escassos; porém, quando ocorrem, os sinais clínicos são característicos de doenças hepáticas (ILHA \& BARROS, 2000). A manifestação de ascite e icterícia no segundo cão descrito confirma essa condição. A ausência de sinais de regeneração de medula óssea no esfregaço sanguíneo desse paciente descartou causas pré-hepáticas de icterícia, como as anemias hemolíticas, conforme descrevem PIEK et al. (2008). Em contrapartida, a ocorrência de icterícia e o aumento das enzimas hepáticas e hipoalbuminemia permitiram concluir se tratar de uma icterícia fundamentalmente de origem hepática, como relatado por HONECKMAN (2003) e WATSON (2004). A respeito das características do líquido cavitário obtido desse paciente, ainda que a insuficiência hepática resulte em hipoalbuminemia e consequentemente na formação de ascite por transudato puro, a formação de liquido peritoneal característico de transudato modificado também pode ocorrer se a hipoalbuminemia for complicada por hipertensão venosa sistêmica (COWELL et al., 1999), como no segundo cão ora relatado.

CERUTI et al. (2001) relataram a presença de ovos de $\boldsymbol{C}$. hepaticum no fígado de $36 \%$ dos ratos ( $R$. norvegicus) analisados, não sendo observados sinais sistêmicos de alterações compatíveis com insuficiência hepática, o que corrobora a hipótese de que os ratos atuam como hospedeiros primários de $\boldsymbol{C}$. hepaticum e como principais reservatórios do parasita em muitas áreas urbanas. Conforme relato dos proprietários, os dois cães aqui descritos tinham contato com ratos nos locais onde viviam, os quais devem ter atuado como fonte de infecção para esses animais.

Desconhece-se a importância clínica da capilariose hepática em caninos; entretanto, o hábito de predação de cães de rua contribui para a disseminação dos ovos, constituindo-se fontes de infecção para os outros animais e para o homem. $\mathrm{O}$ contato de seres humanos com ratos e cães, devido ao caráter zoonótico do parasita, pode ser considerado um risco à saúde pública, especialmente em crianças (ILHA \& BARROS, 2000).

Conclui-se que o diagnóstico de parasitismo por $\boldsymbol{C}$. hepaticum em dois cães em um curto intervalo de tempo sugere probabilidade da existência de maior número de animais infectados, porém sem diagnóstico. O diagnóstico dessa doença é custoso, pois os pacientes podem ser assintomáticos ou apresentar sintomatologia comum a várias desordens hepáticas, além do fato de o diagnóstico ser estabelecido exclusivamente pelo exame histopatológico desse órgão. Vale ressaltar que a principal medida de controle da doença é impedir o contato de cães com roedores, uma vez que estes são os principais hospedeiros do parasita.

\section{REFERÊNCIAS}

CERUTI, R. et al. Capillaria hepatica infection in wild brown rats (Rattus norvegicus) from the urban area of Milan, Italy. Journal of Veterinary Medicine Series B, v.48, n.3, p.235240, 2001. Disponível em: <http:/www.ingentaconnect.com/ content/bsc/jvb/2001/00000048/00000003/art00010>. Acesso em: 7 ago. 2009. doi: 10.1046/j.1439-0450.2001.00436.x

COWELL, R.L et al. Abdominal and thoracic fluid. In: Diagnostic cytology and hematology of the dog and cat. 2.ed. St. Louis: Mosby; 1999. p.142-158.

FARHANG-AZAD, A.; SCHLITTER, D.A. Capillaria hepatica in small mammals collected from Shoa Province, Ethiopia. Journal of Wildlife Diseases, v.14, n.3, p.358-361, 1978.

HONECKMAN, A. Current concepts in the treatment of canine chronic hepatitis. Clinical Techniques in Small Animal Practice, v.18, n.4, p.239-244, 2003. Disponível em: <http:// linkinghub.elsevier.com/retrieve/pii/S1096286703000501>. Acesso em: 7 ago. 2009. doi: 10.1016/S1906-2867(03)00000-0

ILHA, M.R.S; BARROS, C.S.L. Capilariose hepática em cães e gatos: 15 casos. Ciência Rural, v.30, n.4, p.665-669, 2000. Disponível em: <http:/www.scielo.br/scielo.php?pid=S0103$84782000000400018 \&$ script $=$ sci_abstract\&tlng $=\mathrm{pt}>$. Acesso em: 7 ago. 2009 . doi: $10.1590 / \mathrm{S} 0103-84782000000400018$.

PIEK, C.J. et al. Idiophathic immune-mediated hemolytic anemia: treatment outcome and prognostic factors in 149 dogs. Journal of Veterinary Internal Medicine, v.22, n.2, p.366-373, 2008. Disponível em: <http://www3.interscience.wiley.com/journal/ 120715540/abstract?CRETRY $=1 \&$ SRETRY $=0>$. Acesso em: 7 ago. 2009. doi: 10.1111/j.1939-1676.2008.0060.x.

REDROBE, S.P.; PATTERSON-KANE, J.C. Calodium hepaticum (syn. Capillaria hepatica) in captive rodents in a zoological garden. Journal of Comparative Pathology, v.133, p.73-76, 2005. Disponível em: <http://www.sciencedirect.com/ science?_ob=ArticleURL\&_udi=B6WHW-4G63JD3-

Ciência Rural, v.39, n.9, dez, 2009. 
$4 \&$ \&user $=10 \&$ rdoc $=1 \&$ fmt $=\&$ \&orig $=$ search $\&$ sort $=$ d \&_docanchor $=\&$ vi e w $=\mathrm{c} \&$ \& a c c t $=$ C 00000502

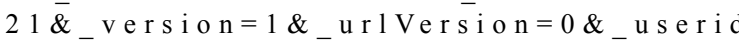
$=10 \& \mathrm{md} 5=\mathrm{fd} 7 \mathrm{f} 078 \mathrm{e} 26 \mathrm{df} 58024550 \mathrm{~b} 428 \mathrm{f} 05 \mathrm{bfe} 52 />$. Acesso em: 7 ago. 2009. doi: 10.1016/j.jcpa.2005.01.013

RUAS, J.L. et al. Infecção por Capillaria hepática em carnívoros silvestres (Lycalopex gymnocercus e Cerdocyon thous) na região sul do Rio Grande do Sul. Arquivos do Instituto Biológico, São Paulo, v.70, n.2, p.127-130, 2003.

SANTOS, A.B. et al. Pathogenesis of hepatic septal fibrosis associated with Capillaria hepatica infection of rats. Revista da Sociedade Brasileira de Medicina Tropical, v.34, n.6, p.503-506, 2001 .

SAWAMURA, R. et al. Hepatic capillariasis in children: report of 3 cases in Brazil. American Journal of Tropical Medicine and Hygiene, v.61, n.4, p. 642-647, 1999.
SOLOMON, G.B.; HANDLEY, C.O.Jr. Capiliaria hepatica (Brancroft, 1893) in Appalachian Mammals. Journal Parasitology, v.57, n.5, p.1142-1144, 1971.

TABOADA, J.; DIMSKI, D.S. Hepatic encephalopathy: clinical signs, pathogenesis, and treatment. Veterinary Clinics of North America: Small Animal Practice, v.25, n.2, p.337$355,1995$.

WATSON, P.J. Chronic hepatitis in dogs: a review of current understanding of the aetiology, progression, and treatment. Veterinary Journal, v.167, p.228-241, 2004. Disponível em: <http:// www.sciencedirect.com/science? ob=ArticleURL\&_udi=B6WXN494C7K4-6\&_user $=10 \& \_r d o c=1 \& \_\mathrm{fmt}=$ \&_orig $=$ search $\&$ sort $=\mathrm{d}$ \&_docanchor $=\&$ view $=\mathrm{c} \&$ _acct $=\mathrm{C} 000050221 \&$ _version $=1 \&$ u r 1 Ve r s i o n $=0 \&$ u s e r i d $=10 \&$ m d $5=$ d 7 c6d0c8c38de3fb69e6412919ff4262>. Acesso em: 7 ago. 2009. doi: 10.1016/S1090-0233(03)00118-7. 